\title{
A UNIQUENESS CRITERION IN THE MULTIVARIATE MOMENT PROBLEM
}

\author{
MIHAI PUTINAR and FLORIAN-HORIA VASILESCU*
}

\begin{abstract}
A determinacy criterion for the multivariate Hamburger moment problem is derived from a recent existence by extension result, [10].
\end{abstract}

\section{Introduction}

The present note is a companion to the article [10]. By exploiting an existence result of [10] we derive below a uniqueness criterion for Hamburger's moment problem in any number of dimensions. Typically, the known determinacy criteria are stated in terms of density of polynomials in certain weighted $L^{p}$ norms, cf. [3], [7], [12]. A notable exception is the Carleman type condition of [5]. We propose below a numerical sufficient condition of determinacy, completely expressible in terms of some associated orthogonal polynomails. We follow the path via a variational problem first studied by M. Riesz, [11].

First let us fix some notation. Let $d$ be a positive integer and let $x=$ $\left(x_{1}, x_{2}, \ldots, x_{d}\right)$ be the coordinates in $\mathrm{R}^{d}$. When embedding (naturally) $\mathrm{R}^{d}$ into $\mathrm{C}^{d}$ we will denote by $z=\left(z_{1}, z_{2}, \ldots, z_{d}\right)$ the complex coordinates. We put $z \cdot z=z_{1}^{2}+z_{2}^{2}+\cdots+z_{d}^{2}$, so that the euclidean norm of the vector $x$ is $|x|=\sqrt{x \cdot x}$. The algebra of polynomials in the indeterminates $x$ will be denoted by $\mathrm{R}[x]$, in the case of real coefficients, and by $\mathrm{C}[z]$ when allowing complex coefficients. For a fixed positive integer $n$, the space of polynomials of degree less or equal than $n$ will be denoted by $\mathrm{R}_{n}[x]$, respectively $\mathrm{C}_{n}[z]$. Whenever it will be necessary, the domain of the polynomial map associated to an element $p \in \mathbf{R}[x]$ will automatically be extended to $\mathrm{C}^{d}$. Throughout this note we denote $\mathbf{N}=\{0,1,2,3, \ldots\}$.

Let $\mu$ be a positive, rapidly decreasing at infinity measure on $\mathrm{R}^{d}$, and let $\mathbf{a}=\left(a_{\alpha}\right)_{\alpha \in \mathbb{N}^{d}}$ be the corresponding moment sequence:

$$
a_{\alpha}=\int_{\mathrm{R}^{d}} x^{\alpha} d \mu(x), \quad \alpha \in \mathrm{N}^{d} .
$$

\footnotetext{
* Partially supported by the National Science Foundation Grant DMS 9800666.

Received October 4, 2000.
} 
Associated solely to the moment sequence is the integration functional:

$$
L(p)=\int_{\mathrm{R}^{d}} p d \mu, \quad p \in \mathrm{R}[x] .
$$

First we recall some basic facts in dimension $d=1$. Let $n$ be a positive integer, and let us consider (after Riesz [11]) the variational problem:

$$
\rho_{n}=\min \left\{L\left(p^{2}\right) ; p \in \mathbf{R}_{n}[x],|p( \pm i)|=1\right\} .
$$

The sequence $\rho_{n}$ is obviously decreasing and the limit $\rho=\lim _{n \rightarrow \infty} \rho_{n}$ is equal to zero if and only if the initial moment problem is determinate (that is, in our notation, $\mu$ is the unique measure with moments a). The real numbers $\rho_{n}$ are the radii of a decreasing set of disks in the plane, representing the values (at $z=i$ ) of the diagonal Padé approximants of the Cauchy transform of the measure $\mu$, see [1] for full details. Most of the uniqueness criteria in the theory of moments in one variable are related to estimates, in different terms, of the limit radius $\rho$.

Since the relation (1) refers to real polynomials $p$, we can obviously replace the condition $|p( \pm i)|=1$ by $|p(i)|=1$. Also, we recall that the numbers $\pm i$ are not privileged; they can be replaced by any pair $\alpha, \bar{\alpha}$ with $\alpha \notin \mathrm{R}$, see [11].

In arbitrary dimension $d \geq 1$ we can define an analogous quantity:

$$
\rho_{n}=\min \left\{L\left(p^{2}\right) ; p \in \mathbf{R}_{n}[x],|p(z)|=1 \text { for } z \cdot z+1=0\right\},
$$

and set $\rho=\lim _{n \rightarrow \infty} \rho_{n}$.

The aim of the present note is to prove that, in any dimension $d$, if $\rho=0$, then the initial moment problem is determinate. We will show that actually the numbers $\rho_{n}$ are computable, for instance in terms of certain orthogonal polynomials depending only on the moment sequence $\mathbf{a}$.

ACKNOWLEDGEMENTS. This work was completed when the first author was visiting the Mathematics Department at the University of Science and Technology of Lille. It is a pleasure for him to thank this institution for hospitality and support.

\section{Main result}

Throughout this section we use the notation introduced before: $\mathbf{a}$ is the moment sequence of the measure $\mu$ on $\mathrm{R}^{d}, d>1$, with associated integration functional $L$ defined on polynomials, and $\rho=\lim _{n \rightarrow \infty} \rho_{n}$, as in relation (2).

First we note that $\rho_{n}$ can be interpreted as a distance in the norm $\|p\|^{2}=$ $L\left(p^{2}\right), p \in \mathrm{R}[x]$. Indeed, the complex variety $V=\left\{z \in \mathrm{C}^{d} ; z \cdot z=-1\right\}$ is a connected smooth hypersurface in $\mathrm{C}^{d}, d>1$, hence by the maximum modulus 
principle (cf. for instance [8] pp. 118), if a polynomial $p \in \mathrm{C}[z]$ satisfies $|p(z)|=1, z \in V$, then there is a constant $c,|c|=1$ such that $p(z)=c$, $z \in V$. To see that the variety $V$ is connected it is sufficient to decompose a point $z \in V$ into real and imaginary parts: $z=x+i y, x, y \in \mathrm{R}^{d}$, and to remark that the equation of $V$ becomes $|x|^{2}-|y|^{2}+1=0, x \cdot y=0$. Then, we can deform $x$ along its direction to zero ( specifically $t x, t \in[0,1]$ ) and deform correspondingly $y$ to the unit vector $y /|y|$. Thus, $V$ is homotopically equivalent to the unit sphere in $\mathrm{R}^{d}, d>1$, hence it is connected.

Moreover, a standard division argument shows that:

$$
p(z)=c-(1+z \cdot z) q(z), \quad q \in \mathrm{C}[z] .
$$

Indeed, the ideal generated by the polynomial $z \cdot z+1$ is prime in every localization of the polynomial ring $\mathrm{C}[z]$, hence it is prime in $\mathrm{C}[z]$. By Hilbert Nullstellensatz ([8] pp. 404), since the polynomial $p(z)-c$ vanishes on $V$ it can be factored by $1+z \cdot z$.

By takinq real and imaginary parts in the coefficients of $q$ we obtain polynomials $r(z), s(x)$, such that $r(x)=\operatorname{Re} q(x), s(x)=\operatorname{Im} q(x), x \in \mathrm{R}^{d}$. Therefore, since we have started with a real polynomial $p$ we obtain:

$$
p(x)=\operatorname{Re} c-\left(1+|x|^{2}\right) r(x), \quad x \in \mathrm{R}^{d},
$$

and

$$
0=\operatorname{Im} c-\left(1+|x|^{2}\right) s(x), \quad x \in \mathrm{R}^{d} .
$$

But the second condition implies $c \in \mathrm{R}$ and $s(z)=0$, hence $c= \pm 1$. Without loss of generality we can assume henceforth that $c=1$.

In conclusion, for $d>1$ and $n \geq 2$ we have proved the following formula:

$$
\rho_{n}=\min \left\{L\left(|p|^{2}\right) ; p(z)=1-(1+z \cdot z) q(z), q \in \mathrm{C}_{n-2}[z]\right\} .
$$

By decomposing $q(x)=r(x)+i s(x), x \in \mathrm{R}^{d}$, as before in real and imaginary parts, we observe that:

$$
|p(x)|^{2}=\left[1-\left(1+|x|^{2}\right) r(x)\right]^{2}+\left(1+|x|^{2}\right)^{2} s(x)^{2}, \quad x \in \mathrm{R}^{d},
$$

so that the minimum in the above expression of $\rho_{n}$ is indeed attained on real polynomials.

THEOREM 2.1. A moment sequence with invariant $\rho=0$ is determinate.

Proof. As recalled before, the case $d=1$ is classical [11], so we can assume $d>1$, in which situation formula (3) holds. If $\rho=0$, then there exists a sequence of polynomials $q_{n} \in \mathrm{R}[x]$ such that

$$
\lim _{n \rightarrow \infty} L\left(\left[1-\left(1+|x|^{2}\right) q_{n}(x)\right]^{2}\right)=0 .
$$


Assume that $v$ is another positive measure, rapidly decreasing at infinity in $\mathrm{R}^{d}$ and having the same moments $\mathbf{a}$ as $\mu$. Then we have:

$$
\lim _{n \rightarrow \infty}\left\|1-\left(1+|x|^{2}\right) q_{n}(x)\right\|_{2, \mu}=\lim _{n \rightarrow \infty}\left\|1-\left(1+|x|^{2}\right) q_{n}(x)\right\|_{2, v}=0 .
$$

Since the function $\frac{1}{1+|x|^{2}}$ is positive and bounded on $\mathrm{R}^{d}$, we infer:

$$
\lim _{n \rightarrow \infty}\left\|\frac{1}{1+|x|^{2}}-q_{n}(x)\right\|_{2, \mu}=\lim _{n \rightarrow \infty}\left\|\frac{1}{1+|x|^{2}}-q_{n}(x)\right\|_{2, v}=0 .
$$

Let $\alpha \in \mathrm{N}^{d}$ be an arbitrary multi-index and let $m$ be a non-negative integer. Our aim is to prove that:

$$
\int_{\mathrm{R}^{d}} \frac{x^{\alpha}}{\left(1+|x|^{2}\right)^{m}} d \mu(x)=\int_{\mathrm{R}^{d}} \frac{x^{\alpha}}{\left(1+|x|^{2}\right)^{m}} d v(x) .
$$

Then a direct argument, or the main result of [10], can be applied and conclude that $\mu=v$.

We prove relation (4) by induction on $m \geq 0$. The case $m=0$ follows from the assumption that both measures have the same moments. Assume that relation (4) is valid for $m$ replaced by $m-1$. Let $\sigma$ be one of the measures $\mu, v$. Since $\frac{x^{\alpha}}{\left(1+|x|^{2}\right)^{m-1}} \in L^{2}(\sigma)$ and $q_{n}(x) \rightarrow \frac{1}{1+|x|^{2}}$ in $L^{2}(\sigma)$, we obtain $\frac{x^{\alpha} q_{n}(x)}{\left(1+|x|^{2}\right)^{m-1}} \rightarrow \frac{x^{\alpha}}{\left(1+|x|^{2}\right)^{m}}$ in $L^{1}(\sigma)$. But according to the induction hypothesis this implies (4).

Note that in the above proof only the convergence $\left\|q_{n}(x)-\frac{1}{1+|x|^{2}}\right\|_{2, \mu} \longrightarrow 0$ was used. However, this latter condition is not intrinsinc in the moments $\mathbf{a}$.

Since, by formula (3), $\sqrt{\rho}$ is the distance in $L^{2}(\mu)$ between the constant function 1 and the subspace $\left(1+|x|^{2}\right) \mathrm{C}[z]$, we obtain the folowing constructive way of computing this number.

Corollary 2.2. Let $P_{\alpha}(x), \alpha \in \mathrm{N}^{d}$, be a sequence of orthonormal polynomials with respect to the measure $\left(1+|x|^{2}\right)^{2} d \mu(x)$ and define the coefficients:

$$
c_{\alpha}=\int_{\mathrm{R}^{d}} P_{\alpha}(x)\left(1+|x|^{2}\right) d \mu(x), \quad \alpha \in \mathrm{N}^{d} .
$$

Then

$$
\rho=a_{0}^{2}-\sum_{\alpha \in \mathrm{N}^{d}} c_{\alpha}^{2}
$$

We remark that $\rho$ is invariant under the orthogonal group action on $\mathbf{R}^{d}$, and moreover, the condition $\rho=0$ is invariant even under all linear transformations of $\mathrm{R}^{d}$. Also it is easy to remark from Corollary 2.2 that the density of 
polynomials in $L^{2}\left(\left(1+|x|^{2}\right)^{2} d \mu(x)\right)$ implies $\rho=0$ (compare with Fuglede's unltradeterminacy condition [7]).

Another possible way of checking the uniqueness condition $\rho(\mathbf{a})=0$ is through the restriction of the moment sequences to the coordinate axes, as in [9]. To be more specific, let $\mathbf{a}$ be the moment sequence of a positive measure $\mu$ on $\mathbf{R}^{d}$, and let $\mathbf{a}_{j}, 1 \leq j \leq d$, be the induced boundary moment sequences:

$$
\mathbf{a}_{j}(\alpha)=\mathbf{a}\left(0, \ldots, 0, \alpha_{j}, 0, \ldots, 0\right)=\int_{\mathrm{R}^{d}} x_{j}^{\alpha_{j}} d \mu(x), \quad \alpha \in \mathrm{N}^{d} .
$$

Then, according to Theorem 3 of [9], if $\rho\left(\mathbf{a}_{j}\right)=0,1 \leq j \leq d$, then $\rho(\mathbf{a})=0$. Morover, the converse is also true in the case of product measures [9] Theorem 4. However, in general the converse is not valid, as shown by an example also contained in [9].

As expected, the condition $\rho=0$ is not necessary, in general, for the unique determination of the representing measure. We present below such an example, adapted after Schmüdgen [12].

PRoposition 2.3. There exists a determinate moment sequence in two variables with $\rho \neq 0$.

Proof. We closely follow the first example in [12]. Let $\mu$ be a positive measure on the real line which admits all moments and is indeterminate, yet $\mathrm{N}$-extremal. That means the polynomials in one variable are dense in $L^{2}(\mu)$, but there exist other measures with the same moments, see also [11]. We define the measure $v=\left(1+x^{2}\right)^{-1} \mu$, so that $v$ is determinate (because for instance the multiplication by $(x+i)$ on polynomials has dense range in $\left.L^{2}(v)\right)$.

Let $j(x)=\left(\sqrt{2} x, x^{2}\right), x \in \mathrm{R}$, be a fixed embedding of the line into $\mathrm{R}^{2}$, and let $\sigma=j_{*} \nu$ be the image measure, supported by the parabola $2 y=x^{2}$. Then it is easy to see that $\sigma$ is a determinate measure, see [12].

Assume that the invariant $\rho$ vanishes for the measure $\sigma$. This means that there exists a sequence of polynomials $p_{n} \in \mathrm{C}[x, y]$ satisfying:

$$
\left\|\left(1+x^{2}+y^{2}\right) p_{n}-1\right\|_{2, \sigma} \longrightarrow 0 .
$$

This in turn implies:

$$
\left\|\left(1+x^{2}\right)^{2} q_{n}-1\right\|_{2, v} \longrightarrow 0,
$$

where $q_{n}(x)=p\left(\sqrt{2} x, x^{2}\right)$. The last condition is equivalent to:

$$
\left\|(x+i)\left(1+x^{2}\right) q_{n}(x)-\frac{1}{x-i}\right\|_{2, \mu} \longrightarrow 0 .
$$


Let $V$ denote the closure of $(x+i) \mathrm{C}[x]$ in $L^{2}(\mu)$. Relation (7) shows that $\frac{1}{x-i} \in V$. Since the measure $\mu$ is indeterminate, for every $\epsilon>0$ there exists a positive constant $C$ with the property that:

$$
|p(z)| \leq C e^{\epsilon|z|}\|p\|_{2, \mu}, \quad z \in \mathrm{C}, \quad p \in \mathrm{C}[x] .
$$

That is the evaluation at a given point $z \in \mathrm{C}$ is a bounded linear functional on the closure of polynomials, hence on all $L^{2}(\mu)$. Moreover, one can identify in this way $L^{2}(\mu)$ with a Hilbert space of entire functions of exponential type, see [11] or [1]. But this contradicts relation (7), because $\left.\frac{1}{z-i}\right|_{z=-i} \neq 0$, while all elements of the space $V$ vanish at the point $z=-i$.

\section{REFERENCES}

1. Akhiezer, N. I., The classical moment problem and some related questions in analysis, Oliver and Boyd, Edinburgh, 1965.

2. Berg, C., Moment problems and polynomial approximation, preprint 1997.

3. Berg, C. and Christensen, J. P. R., Density questions in the classical theory of moments, Ann Inst. Fourier 31(1981), 99-114.

4. Berg, C. and Thill, M., Rotation invariant moment problems, Acta Math. 167 (1991), 207-227.

5. Cramér, H. and Wold, H., Some theorems on distribution functions, J. London Math. Soc. 11 (1936), 290-294.

6. Devinatz, A., Two parameter moment problems, Duke Math. J. 24 (1957), 481-498.

7. B. Fuglede, The multidimensional moment problem, Exposition. Math. 1 (1983), 47-65.

8. Lojasiewicz, S., Introduction to Complex Analytic Geometry, Birkhäuser, 1991.

9. Petersen, L. C., On the relation between the multidimensional moment problem and the onedimensional moment problem, Math. Scand. 51 (1982), 361-366.

10. Putinar, M. and Vasilescu, F.-H., Solving moment problems by dimensional extension, Ann. of Math. 149 (1999), 1087-1107.

11. Riesz, M., Sur le problème des moments. Troisième Note, Ark. Mat. Fys. 17, 16 (1923), 1-52.

12. Schmüdgen, K., On determinacy notions for the two dimensional moment problem, Ark. Mat. 29 (1991), 277-284.

13. Shohat, J. A. and Tamarkin, J. D., The Problem of Moments, Amer. Math. Soc., Providence, 1943.

14. Vasilescu, F.-H., Hamburger and Stieltjes moment problems in several variables, Trans. Amer. Math. Soc. 354 (2002), 1265-1278.

DEPARTMENT OF MATHEMATICS

UNIVERSITY OF CALIFORNIA

SANTA BARBARA, CA 93106

USA

E-mail: mputinar@math.ucsb.edu
U. F. R. DE MATHÉMATIQUES

UNIVERSITÉ DES SCIENCES ET TECHNOLOGIES DE LILLE

U. M. R. DU C. N. R. S. 8524

59655 VILLENEUVE D'ASCQ CEDEX

FRANCE

E-mail: fhvasil@gat.univ-lille1.fr 